\title{
A note on the abundance conjecture
}

\author{
Tobias Dorsch and Vladimir Lazić
}

\begin{abstract}
We prove that the abundance conjecture for non-uniruled Kawamata log terminal pairs in dimension $n$ implies the abundance conjecture for uniruled Kawamata log terminal pairs in dimension $n$, assuming the Minimal Model Program in lower dimensions.
\end{abstract}

\section{Introduction}

The main outstanding conjecture in the Minimal Model Program (MMP) for projective varieties in characteristic zero is that every Kawamata log terminal (klt) pair $(X, \Delta)$ with $K_{X}+\Delta$ pseudoeffective has a minimal model $\left(Y, \Delta_{Y}\right)$ such that $K_{Y}+\Delta_{Y}$ is semiample. Such a minimal model is called a good model. It is well known that the existence of good models implies the abundance conjecture, which predicts that every minimal model is good.

We say that a pair is uniruled if the underlying variety is so, and similarly for a non-uniruled pair. In this paper, we show that it suffices to prove the aforementioned conjectures for nonuniruled pairs. More precisely, the following are our main results.

Theorem 1.1. Assume the existence of good models for klt pairs in dimensions at most $n-1$.

If the abundance conjecture holds for non-uniruled klt pairs in dimension $n$, then the abundance conjecture holds for uniruled klt pairs in dimension $n$.

Theorem 1.2. Assume the existence of good models for klt pairs in dimensions at most $n-1$.

Then the existence of good models for non-uniruled klt pairs in dimension $n$ implies the existence of good models for uniruled klt pairs in dimension $n$.

Observe that by passing to a terminal modification (see Definition 2.1 and Theorem 2.2), and by using the main result of [BDPP13], Theorems 1.1 and 1.2 show that it suffices to prove the existence of good models and the abundance conjecture for terminal pairs $(X, \Delta)$ with $K_{X}$ pseudoeffective.

The existence of good models for surfaces is classical. For terminal threefolds, minimal models were constructed in [Mor88, Sho86], whereas minimal models of canonical fourfolds exist by [BCHM10, Fuj05]. In higher dimensions, the existence of minimal models for klt pairs of log general type is proved in [HM10, BCHM10], and by different methods in [CL12, CL13], whereas

Received 6 October 2014, accepted in final form 12 January 2015.

2010 Mathematics Subject Classification 14E30.

Keywords: Minimal Model Program, abundance conjecture.

This journal is (C) Foundation Compositio Mathematica 2015. This article is distributed with Open Access under the terms of the Creative Commons Attribution Non-Commercial License, which permits non-commercial reuse, distribution, and reproduction in any medium, provided that the original work is properly cited. For commercial re-use, please contact the Foundation Compositio Mathematica.

The authors were supported by the DFG-Emmy-Noether-Nachwuchsgruppe "Gute Strukturen in der höherdimensionalen birationalen Geometrie". 


\section{A Note ON THE ABUNDANCE CONJECTURE}

abundance holds for such pairs by [Sho86, Kaw85b]. Minimal models for effective klt pairs exist assuming the Minimal Model Program in lower dimensions [Bir11].

The abundance conjecture was proved for terminal threefolds in [Miy87, Miy88b, Miy88a, Kaw92] and extended to log canonical threefold pairs $(X, \Delta)$ in the paper [KMM94]. The proof in [KMM94] proceeds by running a $K_{X}$-MMP with scaling of $\Delta$ which is $\left(K_{X}+\Delta\right)$-trivial, to end up either with a Mori fibre space, or with a model $\left(Y, \Delta_{Y}\right)$ on which $K_{Y}+(1-\varepsilon) \Delta_{Y}$ is nef for every $0 \leqslant \varepsilon \ll 1$. In the first case one is almost immediately done by induction even in higher dimensions, whereas in the second case one uses Chern classes, the geometry of surfaces and the case by case analysis of the numerical Kodaira dimension - the argument follows closely the proof for terminal threefolds. A variation of the first case was implemented in [DHP13], and we recall it in Theorem 3.3 below. However, this does not cover all uniruled pairs, as we explain in Remark 3.4. Here we take a different approach to reduce to the case of smooth varieties with effective canonical class.

We briefly explain the strategy of the proof. If $(X, \Delta)$ is a uniruled klt pair, then by [DHP13, Proposition 8.7], we may assume that the adjoint divisor $K_{X}+\Delta$ is effective. We first show that we may furthermore assume that $X$ is smooth and $\Delta$ is a reduced simple normal crossings divisor, and that there exists an effective $\mathbb{Q}$-divisor $D$ such that $K_{X}+\Delta \sim_{\mathbb{Q}} D$ and the supports of $\Delta$ and $D$ are the same. Then we use ramified covers and $\log$ resolutions to construct a $\log$ smooth pair $\left(W, \Delta_{W}\right)$ and a generically finite morphism $w: W \rightarrow X$ such that $K_{W}$ is an effective divisor - we do this by carefully analysing the behaviour of valuations under finite morphisms. We conclude by the construction of $w$ and by the fact that the Kodaira dimension and the numerical Kodaira dimension are preserved under proper morphisms; see Lemma 2.7.

In fact, our techniques lead to the following main technical result of the paper, which implies Theorems 1.1 and 1.2.

Theorem 1.3. Assume the existence of good models for klt pairs in dimensions at most $n-1$.

If good models exist for log smooth klt pairs $(X, \Delta)$ of dimension $n$ such that the linear system $\left|K_{X}\right|$ is not empty, then good models exist for uniruled klt pairs in dimension $n$.

As a by-product, we obtain in Lemma 3.8 a result which can be viewed as a global version of the index one cover [Rei80, Corollary 1.9] and might be of independent interest.

\section{Notation and previous results}

In this section we gather previous results which will be used in Section 3. We pay special attention to the behaviour of discrepancies under finite morphisms; see Proposition 2.14. This result is known [KM98, Proposition 5.20], but we provide the details for the benefit of the reader.

Throughout the paper we work over $\mathbb{C}$. A pair $(X, \Delta)$ consists of a normal variety $X$ and a Weil $\mathbb{Q}$-divisor $\Delta \geqslant 0$ such that the divisor $K_{X}+\Delta$ is $\mathbb{Q}$-Cartier. Such a pair is log smooth if $X$ is smooth and if the support of $\Delta$ has simple normal crossings. We use extensively singularities of pairs; the standard reference is [KM98]. Unless explicitly stated otherwise, all varieties are normal and projective.

\subsection{Terminal modifications}

Terminal modifications allow us to make the singularities of pairs simpler, by replacing klt by terminal singularities. 


\section{T. Dorsch AND V. LAZIĆ}

Definition 2.1. Let $(X, \Delta)$ be a klt pair. A pair $(Y, \Gamma)$ together with a proper birational morphism $f: Y \rightarrow X$ is a terminal modification of $(X, \Delta)$ if the following hold:

(i) The pair $(Y, \Gamma)$ is terminal.

(ii) The variety $Y$ is $\mathbb{Q}$-factorial.

(iii) $K_{Y}+\Gamma \sim_{\mathbb{Q}} f^{*}\left(K_{X}+\Delta\right)$.

We have the following existence result.

Theorem 2.2. If $(X, \Delta)$ is a klt pair, then a terminal modification of $(X, \Delta)$ exists.

Proof. See [BCHM10, Corollary 1.4.3] and the paragraph after that result.

Recall that a variety $X$ of dimension $n$ is uniruled if there is a dominant rational map $\mathbb{P}^{1} \times Y \rightarrow X$, for some variety $Y$ with $\operatorname{dim} Y=n-1$. This property is preserved in the birational equivalence class of $X$. The following result is fundamental.

TheOrem 2.3. Let $X$ be a projective variety with canonical singularities. Then $X$ is uniruled if and only if $K_{X}$ is not pseudoeffective.

Proof. For manifolds, this is [BDPP13, Corollary 0.3]. The result for varieties with canonical singularities follows immediately.

\subsection{Good models}

We recall the definition of log terminal and good models.

Definition 2.4. Let $X$ and $Y$ be $\mathbb{Q}$-factorial varieties, and let $D$ be a $\mathbb{Q}$-divisor on $X$. A birational contraction $f: X \rightarrow Y$ is a log terminal model for $D$ if $f_{*} D$ is nef and if there exists a resolution $(p, q): W \rightarrow X \times Y$ of the map $f$ such that $p^{*} D=q^{*} f_{*} D+E$, where $E \geqslant 0$ is a $q$-exceptional $\mathbb{Q}$-divisor which contains the proper transform of every $f$-exceptional divisor in its support. If additionally $f_{*} D$ is semiample, then the map $f$ is called a good model for $D$.

Note that if $(X, \Delta)$ is a klt pair, then it has a good model if and only if there exists a Minimal Model Program with scaling of an ample divisor which terminates with a good model of $(X, \Delta)$; see [Lai11, Propositions 2.4 and 2.5].

Theorem 2.5. Assume the existence of good models for klt pairs in dimensions at most $n-1$.

Let $(X, \Delta)$ be a klt pair of dimension $n$ which is projective over a projective variety $Z$ such that $K_{X}+\Delta$ is effective over $Z$. Then $(X, \Delta)$ has a log terminal model over $Z$.

Proof. By [Bir11, Corollary 1.7 and the paragraph after Definition 2.2], it is enough to show that every $\mathbb{Q}$-factorial divisorial log terminal (dlt) pair $(Y, \Gamma)$ of dimension at most $n-1$ such that $K_{Y}+$ $\Gamma$ is pseudoeffective has a minimal model in the sense of Birkar and Shokurov; see [Bir11, Definition 2.1]. To this end, note first that $\kappa\left(Y, K_{Y}+\Gamma\right) \geqslant 0$ by our assumption and by [Gon12, Theorem 1.5] and [FG14, Theorem 5.5]. We conclude by induction and by [Bir11, Corollary 1.7].

Kawamata [Kaw85a] was the first to realise that the numerical Kodaira dimension, in the case of nef divisors, plays a crucial role in the abundance conjecture. The concept was generalised in [Nak04] to the case of pseudoeffective divisors. 


\section{A Note on the ABUndance CONJECTURE}

Definition 2.6. Let $X$ be a smooth projective variety, and let $D$ be a pseudoeffective $\mathbb{Q}$-divisor on $X$. If we denote

$$
\sigma(D, A)=\sup \left\{k \in \mathbb{N} \mid \liminf _{m \rightarrow \infty} h^{0}(X,\lfloor m D\rfloor+A) / m^{k}>0\right\}
$$

for a Cartier divisor $A$ on $X$, then the numerical Kodaira dimension of $D$ is

$$
\kappa_{\sigma}(X, D)=\sup \{\sigma(D, A) \mid A \text { is ample }\} .
$$

If $X$ is a projective variety and if $D$ is a pseudoeffective $\mathbb{Q}$-Cartier $\mathbb{Q}$-divisor on $X$, then we set $\kappa_{\sigma}(X, D)=\kappa_{\sigma}\left(Y, f^{*} D\right)$ for any birational morphism $f: Y \rightarrow X$ from a smooth projective variety $Y$.

The following lemma shows that the function $\kappa_{\sigma}$ behaves similarly to the Kodaira dimension under proper pullbacks.

Lemma 2.7. Let $D$ be a $\mathbb{Q}$-divisor on a $\mathbb{Q}$-factorial variety $X$, and let $f: Y \rightarrow X$ be a proper surjective morphism. Then

$$
\kappa(X, D)=\kappa\left(Y, f^{*} D\right) \quad \text { and } \quad \kappa_{\sigma}(X, D)=\kappa_{\sigma}\left(Y, f^{*} D\right) .
$$

If $f$ is birational and $E$ is an effective $f$-exceptional divisor on $Y$, then

$$
\kappa(X, D)=\kappa\left(Y, f^{*} D+E\right) \quad \text { and } \quad \kappa_{\sigma}(X, D)=\kappa_{\sigma}\left(Y, f^{*} D+E\right) .
$$

Proof. The first three relations are [Nak04, Lemma II.3.11 and Proposition V.2.7(4)]. For the last one, we have $P_{\sigma}\left(f^{*} D+E\right)=P_{\sigma}\left(f^{*} D\right)$ by [GL13, Lemma 2.16]; hence $\kappa_{\sigma}\left(Y, f^{*} D+E\right)=$ $\kappa_{\sigma}\left(Y, f^{*} D\right)$ by [Leh13, Theorem 6.7].

The following result generalises [Kaw85a, Theorem 6.1]; it will be crucial in the proofs in the following section.

Lemma 2.8. Let $(X, \Delta)$ be a klt pair. Then $(X, \Delta)$ has a good model if and only if $\kappa\left(X, K_{X}+\Delta\right)=$ $\kappa_{\sigma}\left(X, K_{X}+\Delta\right)$.

Proof. This is [GL13, Theorem 4.3].

Lemma 2.9. Let $(X, \Delta)$ and $\left(X, \Delta^{\prime}\right)$ be pairs, and assume that there exist $\mathbb{Q}$-divisors $D \geqslant 0$ and $D^{\prime} \geqslant 0$ such that

$$
K_{X}+\Delta \sim_{\mathbb{Q}} D \geqslant 0, \quad K_{X}+\Delta^{\prime} \sim_{\mathbb{Q}} D^{\prime} \geqslant 0 \quad \text { and } \quad \operatorname{Supp} D=\operatorname{Supp} D .
$$

Then

$$
\kappa\left(X, K_{X}+\Delta\right)=\kappa\left(X, K_{X}+\Delta^{\prime}\right) \quad \text { and } \quad \kappa_{\sigma}\left(X, K_{X}+\Delta\right)=\kappa_{\sigma}\left(X, K_{X}+\Delta^{\prime}\right) .
$$

Proof. There exist positive rational numbers $t_{1}$ and $t_{2}$ such that $t_{1} D \leqslant D^{\prime} \leqslant t_{2} D$; hence $\kappa\left(X, t_{1} D\right) \leqslant \kappa\left(X, D^{\prime}\right) \leqslant \kappa\left(X, t_{2} D\right)$. This implies the first equality, and the proof of the second is analogous.

\subsection{Valuations under finite morphisms}

Definition 2.10. A geometric valuation $\Gamma$ on a normal variety $X$ is a valuation on the function field $k(X)$ given by the order of vanishing at the generic point of a prime divisor on some proper birational model $f: Y \rightarrow X$; by abusing notation, we identify $\Gamma$ with the corresponding prime divisor. If $D$ is an $\mathbb{R}$-Cartier divisor on $X$, we use $\operatorname{mult}_{\Gamma} D$ to denote mult $f^{*} D$. The set $f(\Gamma)$ is the centre of $\Gamma$ on $X$ and is denoted by $c_{X}(\Gamma)$. 


\section{T. Dorsch AND V. LAZIĆ}

Remark 2.11. With notation from the definition, let $R$ be a discrete valuation ring with quotient field $k(X)$ which dominates the local ring $\mathcal{O}_{X, c_{X}(\Gamma)} \subseteq k(X)$. Then there exists a morphism Spec $R \rightarrow X$ which sends the generic point of Spec $R$ to the generic point of $X$, and the closed point of Spec $R$ to the generic point of $c_{X}(\Gamma)$; see [Har77, Lemma II.4.4]. In particular, this holds if $R=\mathcal{O}_{Y, \Gamma}$.

Remark 2.12. Let $X$ be a normal variety, and let $(R, m)$ be a discrete valuation ring such that the quotient field of $R$ is $k(X)$. Assume that there is a morphism $\operatorname{Spec} R \rightarrow X$ which sends the generic point of $\operatorname{Spec} R$ to the generic point of $X$. Assume $\operatorname{trdeg}_{\mathbb{C}}(R / m)=\operatorname{dim} X-1$. Then by a lemma of Zariski [KM98, Lemma 2.45], the corresponding valuation is a geometric valuation on $X$.

We first prove an easy algebraic result that we use in the proof of Proposition 2.14.

Lemma 2.13. Let $k \subseteq K$ be an algebraic extension of fields. Let $\left(B, m_{B}\right)$ be a discrete valuation ring with the quotient field $K$, and let $A=B \cap k$ and $m_{A}=m_{B} \cap k$. Then $\left(A, m_{A}\right)$ is a discrete valuation ring with the quotient field $k$ such that the field extension $A / m_{A} \subseteq B / m_{B}$ is algebraic.

Proof. Let $\nu: K \rightarrow \mathbb{Z} \cup\{\infty\}$ be the valuation function corresponding to $\left(B, m_{B}\right)$. Then $A=$ $\{a \in k \mid \nu(a) \geqslant 0\}$ and $m_{A}=\{a \in k \mid \nu(a)>0\}$, and it is immediate that $k$ is the quotient field of $A$. Let $b \in B$ and denote $\bar{b}=b+m_{B} \in B / m_{B}$. Then there is a polynomial

$$
p=T^{n}+r_{n-1} T^{n-1}+\cdots+r_{0} \in k[T]
$$

such that $p(b)=0$. Fix $j \in\{0, \ldots, n-1\}$ such that $\nu\left(r_{j}\right) \leqslant \nu\left(r_{i}\right)$ for all $i$. If $\nu\left(r_{j}\right) \geqslant 0$, then $p \in A[T]$ and $\bar{b}$ is algebraic over $A / m_{A}$. If $\nu\left(r_{j}\right)<0$, then $r_{j}^{-1} \in m_{A}$ and $\nu\left(r_{j}^{-1} r_{i}\right) \geqslant 0$ for all $i$. Therefore,

$$
\bar{p}=r_{j}^{-1} p \quad \bmod m_{A} \in\left(A / m_{A}\right)[T]
$$

is a non-zero polynomial such that $\bar{p}(\bar{b})=0$, which proves the last claim. It remains to show $m_{A} \neq\{0\}$. Fix $b \in B$ with $\nu(b)>0$, and let

$$
p=a_{n} T^{n}+a_{n-1} T^{n-1}+\cdots+a_{0} \in A[T]
$$

be a polynomial of minimal degree such that $p(b)=0$, so that, in particular, $a_{0} \neq 0$. Then we have

$$
0<\nu(b) \leqslant \nu\left(b\left(a_{n} b^{n-1}+a_{n-1} b^{n-2}+\cdots+a_{1}\right)\right)=\nu\left(-a_{0}\right)
$$

hence $a_{0} \in m_{A}$.

Proposition 2.14. Let $\pi: X^{\prime} \rightarrow X$ be a finite morphism of degree $m$ between normal varieties, let $\Delta$ be a $\mathbb{Q}$-divisor on $X$ such that $(X, \Delta)$ is a pair, and let $\Delta^{\prime}$ be a $\mathbb{Q}$-divisor on $X^{\prime}$ such that $K_{X^{\prime}}+\Delta^{\prime}=\pi^{*}\left(K_{X}+\Delta\right)$.

(i) For every geometric valuation $E^{\prime}$ over $X^{\prime}$ there exist a geometric valuation $E$ over $X$ and an integer $1 \leqslant r \leqslant m$ such that $\pi\left(c_{X^{\prime}}\left(E^{\prime}\right)\right)=c_{X}(E)$ and

$$
a\left(E^{\prime}, X^{\prime}, \Delta^{\prime}\right)+1=r(a(E, X, \Delta)+1) .
$$

(ii) For every geometric valuation $E$ over $X$ there exist a geometric valuation $E^{\prime}$ over $X^{\prime}$ and an integer $1 \leqslant r \leqslant m$ such that $\pi\left(c_{X^{\prime}}\left(E^{\prime}\right)\right)=c_{X}(E)$ and

$$
a\left(E^{\prime}, X^{\prime}, \Delta^{\prime}\right)+1=r(a(E, X, \Delta)+1) .
$$

In particular, the pair $(X, \Delta)$ is log canonical (respectively, klt) if and only if the pair $\left(X^{\prime}, \Delta^{\prime}\right)$ is $\log$ canonical (respectively, klt). 


\section{A Note on the ABUndance CONJECTURE}

Proof. This is [KM98, Proposition 5.20], and in the following we reproduce the proof with more details.

We claim that in both case (i) and case (ii) there is a commutative diagram

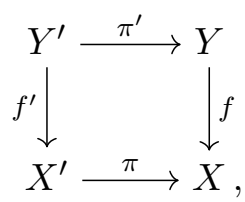

where $f$ and $f^{\prime}$ are birational morphisms, $\pi^{\prime}$ is finite, and there are prime divisors $E \subseteq Y$ and $E^{\prime} \subseteq Y^{\prime}$ such that $\pi^{\prime}\left(E^{\prime}\right)=E$. The claim immediately implies the proposition. Indeed, let $r=\operatorname{mult}_{E^{\prime}}\left(\pi^{\prime}\right)^{*} E$. Then locally around the generic point of $E^{\prime}$ we have

$$
\begin{aligned}
K_{Y^{\prime}}-(r-1) E^{\prime} & =\left(\pi^{\prime}\right)^{*} K_{Y} \sim_{\mathbb{Q}}\left(\pi^{\prime}\right)^{*}\left(f^{*}\left(K_{X}+\Delta\right)+a(E, X, \Delta) \cdot E\right) \\
& =\left(f^{\prime}\right)^{*}\left(K_{X}^{\prime}+\Delta^{\prime}\right)+r \cdot a(E, X, \Delta) \cdot E^{\prime} \\
& \sim_{\mathbb{Q}} K_{Y^{\prime}}-a\left(E^{\prime}, X^{\prime}, \Delta^{\prime}\right) \cdot E^{\prime}+r \cdot a(E, X, \Delta) \cdot E^{\prime} ;
\end{aligned}
$$

hence cases (i) and (ii) follow.

To see the claim in case (ii), let $f: Y \rightarrow X$ be a birational morphism such that $E \subseteq Y$ is a prime divisor, and let $Y^{\prime}$ be a component of the normalisation of the fibre product $X^{\prime} \times_{X} Y$ that maps onto $Y$. Then we obtain diagram (2.1), and since $\pi^{\prime}$ is surjective, there is a prime divisor $E^{\prime} \subseteq Y^{\prime}$ with $\pi^{\prime}\left(E^{\prime}\right)=E$.

In case (i), let $\left(R^{\prime}, m_{R^{\prime}}\right)$ be the discrete valuation ring corresponding to the valuation $E^{\prime}$, and let $R=R^{\prime} \cap k(X)$ and $m_{R}=m_{R^{\prime}} \cap k(X)$. Since $k(X) \subseteq k\left(X^{\prime}\right)$ is an algebraic extension of fields, $R$ is a discrete valuation ring with quotient field $k(X)$ such that $\operatorname{trdeg} \operatorname{Cog}_{\mathbb{C}}\left(R / m_{R}\right)=\operatorname{dim} X-1$ by Lemma 2.13. If $E$ is the corresponding discrete valuation, then $E$ is a divisorial valuation by Remark 2.12. By Remark 2.11, there is a morphism $\rho^{\prime}:$ Spec $R^{\prime} \rightarrow X^{\prime}$ which sends the generic point of Spec $R^{\prime}$ to the generic point of $X^{\prime}$, and the closed point of $\operatorname{Spec} R^{\prime}$ to the generic point $\eta^{\prime}$ of $c_{X^{\prime}}\left(E^{\prime}\right)$. If $\eta=\pi\left(\eta^{\prime}\right)$, then

$$
\mathcal{O}_{X, \eta} \subseteq \mathcal{O}_{X^{\prime}, \eta^{\prime}} \cap k(X) \subseteq R^{\prime} \cap k(X)=R
$$

hence by Remark 2.11 there is a morphism $\rho: \operatorname{Spec} R \rightarrow X$ which sends the generic point of Spec $R$ to the generic point of $X$, and the closed point of $\operatorname{Spec} R$ to $\eta$.

Let $f: Y \rightarrow X$ be a birational morphism such that $E$ is a divisor on $Y$, and denote by $X^{\prime}$ a component of the normalisation of the fibre product $X^{\prime} \times_{X} Y$ that maps onto $Y$, so that we have diagram (2.1). By the valuative criterion of properness, we have the diagram

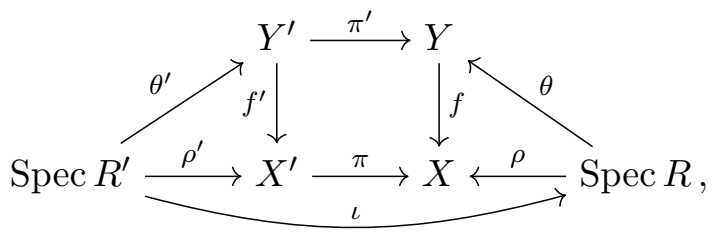

where $\iota: \operatorname{Spec} R^{\prime} \rightarrow \operatorname{Spec} R$ is the morphism induced by the inclusion $R \subseteq R^{\prime}$. Since $f$ is separated, we have $\pi^{\prime} \circ \theta^{\prime}=\theta \circ \iota$, which just says that $E^{\prime}$ is a prime divisor on $Y^{\prime}$ such that $\pi^{\prime}\left(E^{\prime}\right)=E$. 


\section{T. Dorsch ANd V. LAZIĆ}

\section{Good models for uniruled pairs}

Lemma 3.1. Let $(X, \Delta)$ be a pair, and let $f: X \rightarrow Y$ be a birational contraction to a normal projective variety such that $K_{Y}+f_{*} \Delta$ is $\mathbb{Q}$-Cartier. Then

$$
\kappa_{\sigma}\left(X, K_{X}+\Delta\right) \leqslant \kappa_{\sigma}\left(Y, K_{Y}+f_{*} \Delta\right) .
$$

Proof. Let $(p, q): W \rightarrow X \times Y$ be a resolution of the map $f$. Write

$$
K_{W}+\Delta_{W} \sim_{\mathbb{Q}} p^{*}\left(K_{X}+\Delta\right)+E \text { and } K_{W}+\Delta_{W}^{\prime} \sim_{\mathbb{Q}} q^{*}\left(K_{Y}+f_{*} \Delta\right)+E^{\prime},
$$

where $\Delta_{W} \geqslant 0$ and $E \geqslant 0$ have no common components, and $\Delta_{W}^{\prime} \geqslant 0$ and $E^{\prime} \geqslant 0$ have no common components. Since $f$ is a contraction, the divisor $\Delta_{W}-\Delta_{W}^{\prime}$ is q-exceptional, and there are effective $q$-exceptional $\mathbb{Q}$-divisors $E^{+}$and $E^{-}$such that $\Delta_{W}-\Delta_{W}^{\prime}=E^{+}-E^{-}$. Therefore,

$$
K_{W}+\Delta_{W}+E^{-}=K_{W}+\Delta_{W}^{\prime}+E^{+} \sim_{\mathbb{Q}} q^{*}\left(K_{Y}+f_{*} \Delta\right)+E^{\prime}+E^{+} ;
$$

hence $\kappa_{\sigma}\left(W, K_{W}+\Delta_{W}+E^{-}\right)=\kappa_{\sigma}\left(Y, K_{Y}+f_{*} \Delta\right)$ by Lemma 2.7. We conclude since $\kappa_{\sigma}\left(X, K_{X}+\right.$ $\Delta)=\kappa_{\sigma}\left(W, K_{W}+\Delta_{W}\right) \leqslant \kappa_{\sigma}\left(W, K_{W}+\Delta_{W}+E^{-}\right)$by Lemma 2.7.

Definition 3.2. Let $(X, \Delta)$ be a klt pair. Let $G$ be an effective $\mathbb{Q}$-Cartier $\mathbb{Q}$-divisor such that $K_{X}+\Delta+G$ is pseudoeffective. Then the pseudoeffective threshold $\tau(X, \Delta ; G)$ is defined as

$$
\tau(X, \Delta ; G)=\min \left\{t \in \mathbb{R} \mid K_{X}+\Delta+t G \text { is pseudoeffective }\right\} .
$$

Theorem 3.3. Assume the existence of good models for klt pairs in dimensions at most $n-1$.

Let $(X, \Delta)$ be a klt pair of dimension $n$. Let $G$ be an effective $\mathbb{Q}$-Cartier $\mathbb{Q}$-divisor such that $(X, \Delta+G)$ is klt and $K_{X}+\Delta+G$ is pseudoeffective. Assume that $K_{X}+\Delta$ is not pseudoeffective, in other words, that $\tau=\tau(X, \Delta ; G)>0$.

Then $\tau \in \mathbb{Q}$, and there exists a good model of $(X, \Delta+\tau G)$. In particular,

$$
\kappa\left(X, K_{X}+\Delta+\tau G\right) \geqslant 0 .
$$

Proof. We follow closely the proofs of [DHP13, Proposition 8.7 and Theorem 8.8], which show that $\tau \in \mathbb{Q}$ and $\kappa\left(X, K_{X}+\Delta+\tau G\right) \geqslant 0$. Fix an ample divisor $A$ on $X$. For any rational number $0 \leqslant x \leqslant \tau$, let $y_{x}=\tau(X, \Delta+x G ; A)$. Note that $y_{\tau}=0$ and that $y_{x}$ is a positive rational number for $0 \leqslant x<\tau$-rationality follows from [BCHM10, Corollary 1.1.7] and positivity from the fact that $K_{X}+\Delta+x G$ is not pseudoeffective when $x<\tau$.

Let $\left(x_{i}\right)$ be an increasing sequence of non-negative rational numbers such that $\lim _{i \rightarrow \infty} x_{i}=\tau$, and denote $y_{i}=y_{x_{i}}$. Fix $i$, let $f_{i}: X \rightarrow Y_{i}$ be the $\left(K_{X}+\Delta+x_{i} G\right)$-MMP with scaling of $A$, and denote by $\Delta_{i}, G_{i}$ and $A_{i}$ the proper transforms on $Y_{i}$ of $\Delta, G$ and $A$, respectively. By [BCHM10, Corollary 1.3.3], there is an extremal contraction $g_{i}: Y_{i} \rightarrow Z_{i}$ of fibre type such that

$$
K_{Y_{i}}+\Delta_{i}+x_{i} G_{i}+y_{i} A_{i} \equiv_{g_{i}} 0 .
$$

Let $E_{j}$ be effective divisors on $Y_{i}$ whose classes converge to the class of $K_{Y_{i}}+\Delta_{i}+\tau G_{i}$ in $N^{1}\left(Y_{i}\right)_{\mathbb{R}}$, and let $C$ be a curve on $Y_{i}$ which does not belong to $\bigcup \operatorname{Supp} E_{j}$ and is contracted by $g_{i}$. Then

$$
\left(K_{Y_{i}}+\Delta_{i}+\tau G_{i}\right) \cdot C \geqslant 0 \text { and }\left(K_{Y_{i}}+\Delta_{i}+x_{i} G_{i}+y_{i} A_{i}\right) \cdot C=0 .
$$

Therefore, there exists a rational number $\eta_{i} \in\left(x_{i}, \tau\right]$ such that $\left(K_{Y_{i}}+\Delta_{i}+\eta_{i} G_{i}\right) \cdot C=0$; hence

$$
K_{Y_{i}}+\Delta_{i}+\eta_{i} G_{i} \equiv_{g_{i}} 0,
$$

since all contracted curves are numerically proportional. In particular, if $F_{i}$ is a general fibre of $g_{i}$, and $\Delta_{F_{i}}=\left.\Delta_{i}\right|_{F_{i}}$ and $G_{F_{i}}=\left.G_{i}\right|_{F_{i}}$, then

$$
K_{F_{i}}+\Delta_{F_{i}}+\eta_{i} G_{F_{i}} \equiv 0 .
$$


Denoting

$$
\tau_{i}=\max \left\{t \in \mathbb{R} \mid K_{F_{i}}+\Delta_{F_{i}}+t G_{F_{i}} \text { is log canonical }\right\},
$$

we have $x_{i} \leqslant \tau_{i}$ since $K_{F_{i}}+\Delta_{F_{i}}+x_{i} G_{F_{i}}$ is $\log$ canonical for every $i$. If $K_{F_{i}}+\Delta_{F_{i}}+\tau G_{F_{i}}$ is not $\log$ canonical for infinitely many $i$, then after passing to a subsequence we may assume $\tau_{i}<\tau$ for all $i$. Moreover, since $x_{i} \leqslant \tau_{i}$ and $\lim x_{i}=\tau$, we may assume that the sequence $\left(\tau_{i}\right)$ is strictly increasing, which contradicts [HMX14, Theorem 1.1]. Therefore, $K_{F_{i}}+\Delta_{F_{i}}+\tau G_{F_{i}}$ is $\log$ canonical for $i \gg 0$, and then [HMX14, Theorem 1.5] implies that the sequence $\left(\eta_{i}\right)$ is eventually constant; hence $\eta_{i}=\tau$ for $i \gg 0$. In particular, $\tau \in \mathbb{Q}$.

Now, for the rest of the proof fix any such $i \gg 0$ for which $\eta_{i}=\tau$, and let $(p, q): W \rightarrow X \times Y_{i}$ be a resolution of the map $f_{i}$ :

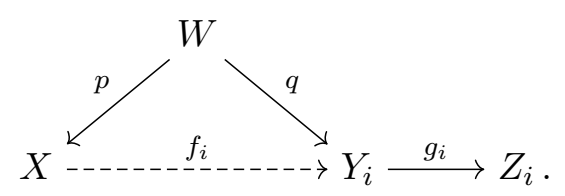

We may write

$$
K_{W}+\Delta_{W} \sim_{\mathbb{Q}} p^{*}\left(K_{X}+\Delta+\tau G\right)+E,
$$

where $\Delta_{W}$ and $E$ are effective $\mathbb{Q}$-divisors without common components. We want to prove that $(X, \Delta+\tau G)$ has a good model; hence by Lemmas 2.7 and 2.8 , it is enough to show

$$
\kappa\left(W, K_{W}+\Delta_{W}\right)=\kappa_{\sigma}\left(W, K_{W}+\Delta_{W}\right) .
$$

If we denote $F_{W}=q^{-1}\left(F_{i}\right) \subseteq W$, then $\left(\left.q\right|_{F_{W}}\right)_{*}\left(K_{F_{W}}+\left.\Delta_{W}\right|_{F_{W}}\right)=K_{F_{i}}+\Delta_{F_{i}}+\tau G_{F_{i}}$; hence by Lemma 3.1 and by (3.1),

$$
\kappa_{\sigma}\left(F_{W}, K_{F_{W}}+\left.\Delta_{W}\right|_{F_{W}}\right) \leqslant \kappa_{\sigma}\left(F_{i}, K_{F_{i}}+\Delta_{F_{i}}+\tau G_{F_{i}}\right)=0 .
$$

When $\operatorname{dim} Z_{i}=0$, we have $F_{W}=W$ and (3.3) implies (3.2) by [Nak04, Corollary V.4.9].

When $\operatorname{dim} Z_{i}>0$, the divisor $K_{W}+\Delta_{W}$ is effective over $Z_{i}$ by induction on the dimension and by [BCHM10, Lemma 3.2.1]. By Theorem 2.5 and by [Fuj11, Theorem 1.1], there exists a good model $\left(W, \Delta_{W}\right) \rightarrow\left(W_{\min }, \Delta_{\min }\right)$ of $\left(W, \Delta_{W}\right)$ over $Z_{i}$. Let $\varphi: W_{\min } \rightarrow W_{\text {can }}$ be the corresponding fibration to the canonical model of $\left(W, \Delta_{W}\right)$ over $Z_{i}$. Since $K_{W}+\Delta_{W}$ is not big over $Z_{i}$ by (3.3), we have $\operatorname{dim} W_{\text {can }}<\operatorname{dim} X$. By [Amb05, Theorem 0.2], there exists a divisor $\Delta_{\text {can }}$ on $W_{\text {can }}$ such that the pair $\left(W_{\text {can }}, \Delta_{\text {can }}\right)$ is klt and

$$
K_{W_{\min }}+\Delta_{\min } \sim_{\mathbb{Q}} \varphi^{*}\left(K_{W_{\text {can }}}+\Delta_{\text {can }}\right) .
$$

Since we assume the existence of good models for klt pairs in dimensions at most $n-1$, we have $\kappa\left(W_{\text {can }}, K_{W_{\text {can }}}+\Delta_{\text {can }}\right)=\kappa_{\sigma}\left(W_{\text {can }}, K_{W_{\text {can }}}+\Delta_{\text {can }}\right)$ by Lemma 2.8, and hence (3.2) holds by Lemma 2.7, which concludes the proof.

Remark 3.4. Let $(X, \Delta)$ be a uniruled klt pair such that $K_{X}$ is not pseudoeffective and $K_{X}+\Delta$ is pseudoeffective. A natural strategy to construct a good model of $(X, \Delta)$ is to run a $\left(K_{X}+\tau \Delta\right)$ MMP, where $\tau=\tau(X, 0 ; \Delta)$, and which we know terminates with a good model $\left(Y, \Delta_{Y}\right)$ by Theorem 3.3. The main problem is that this MMP does not preserve sections of $K_{X}+\Delta$. An instructive example is when $K_{X} \sim_{\mathbb{Q}}-\tau \Delta$, where $\Delta$ is nef and not big, and for instance $\rho(X)=2$. Then one might want to run the $\left(K_{X}+(\tau-\varepsilon) \Delta\right)$-MMP with scaling of an ample divisor $A$, where $0<\varepsilon \ll 1$. If the nef cone $\operatorname{Nef}(X)$ is not equal to the pseudoeffective cone $\overline{\operatorname{Eff}}(X)$, then this MMP ends up with a model on which the proper transform of $K_{X}+\Delta$ is ample, regardless of the Kodaira dimension of $K_{X}+\Delta$. 


\section{T. Dorsch AND V. LAZIĆ}

Theorem 3.5. Assume the existence of good models for klt pairs in dimensions at most $n-1$, and the existence of good models for $\log$ smooth klt pairs $(X, \Delta)$ in dimension $n$ such that $\left|K_{X}\right| \neq \emptyset$.

Let $(X, \Delta)$ be a $\log$ smooth $\log$ canonical pair of dimension $n$ and assume that there exists a $\mathbb{Q}$-divisor $D \geqslant 0$ such that $K_{X}+\Delta \sim_{\mathbb{Q}} D$ and $\operatorname{Supp} \Delta=\operatorname{Supp} D$. Then

$$
\kappa\left(X, K_{X}+\Delta\right)=\kappa_{\sigma}\left(X, K_{X}+\Delta\right) .
$$

Proof. Replacing $\Delta$ by $\lceil\Delta\rceil$, by Lemma 2.9 we may assume that the divisor $\Delta$ is reduced. In the course of the proof, we construct a tower of proper maps

$$
\left(T, \Delta_{T}\right) \stackrel{\mu}{\longrightarrow}\left(W, \Delta_{W}\right) \stackrel{g}{\longrightarrow}\left(X^{\prime}, \Delta_{X^{\prime}}\right) \stackrel{\pi}{\longrightarrow}(X, \Delta),
$$

where $\pi$ and $\mu$ are finite and $g$ is birational, such that for each $\mathcal{X} \in\left\{T, W, X^{\prime}\right\}$ we have

$$
\kappa\left(\mathcal{X}, K_{\mathcal{X}}+\Delta_{\mathcal{X}}\right)=\kappa\left(X, K_{X}+\Delta\right) \quad \text { and } \quad \kappa_{\sigma}\left(\mathcal{X}, K_{\mathcal{X}}+\Delta_{\mathcal{X}}\right)=\kappa_{\sigma}\left(X, K_{X}+\Delta\right) .
$$

The pair $\left(T, \Delta_{T}\right)$ will be a $\log$ smooth klt pair with $\left|K_{T}\right| \neq \emptyset$, which allows us to conclude.

Let $m$ be the smallest positive integer such that $m\left(K_{X}+\Delta\right) \sim m D$, and denote $G=m D$. Let $\pi: X^{\prime} \rightarrow X$ be the normalisation of the corresponding $m$-fold cyclic covering ramified along $G$. Note that $X^{\prime}$ is irreducible by [EV92, Lemma 3.15(a)] since $m$ is minimal. Then there exists an effective Cartier divisor $G^{\prime}$ on $X^{\prime}$ such that

$$
\pi^{*} G=m G^{\prime} \quad \text { and } \quad \pi^{*}\left(K_{X}+\Delta\right) \sim G^{\prime} .
$$

Let $\Delta^{\prime}=\left(G^{\prime}\right)_{\text {red }}$. By the Hurwitz formula, we have

$$
K_{X^{\prime}}+\Delta^{\prime}=\pi^{*}\left(K_{X}+\Delta\right),
$$

and the pair $\left(X^{\prime}, \Delta^{\prime}\right)$ is $\log$ canonical by Proposition 2.14. We have

$$
\kappa\left(X, K_{X}+\Delta\right)=\kappa\left(X^{\prime}, K_{X^{\prime}}+\Delta^{\prime}\right) \quad \text { and } \quad \kappa_{\sigma}\left(X, K_{X}+\Delta\right)=\kappa_{\sigma}\left(X^{\prime}, K_{X^{\prime}}+\Delta^{\prime}\right)
$$

by Lemma 2.7. We claim that for every geometric valuation $E^{\prime}$ over $X^{\prime}$ we have $a\left(E^{\prime}, X^{\prime}, \Delta^{\prime}\right) \in \mathbb{Z}$. To prove this, let $E^{\prime}$ be a geometric valuation over $X^{\prime}$. Then by Proposition 2.14, there exist a geometric valuation $E$ over $X$ and an integer $1 \leqslant r \leqslant m$ such that

$$
a\left(E^{\prime}, X^{\prime}, \Delta^{\prime}\right)+1=r(a(E, X, \Delta)+1) .
$$

Since $(X, \Delta)$ is $\log$ smooth and $\Delta$ is reduced, we have $a(E, X, \Delta) \in \mathbb{Z}$, which together with (3.4) implies the claim.

Now, if $g: W \rightarrow X^{\prime}$ is a log resolution of the pair $\left(X^{\prime}, \Delta^{\prime}\right)$, by the claim we may write

$$
K_{W}+\Delta_{W} \sim_{\mathbb{Q}} g^{*}\left(K_{X^{\prime}}+\Delta^{\prime}\right)+E_{W} \sim g^{*} G^{\prime}+E_{W}
$$

where $\Delta_{W}$ and $E_{W}$ are effective integral divisors with no common components. Then

$$
\kappa\left(X, K_{X}+\Delta\right)=\kappa\left(W, K_{W}+\Delta_{W}\right) \quad \text { and } \quad \kappa_{\sigma}\left(X, K_{X}+\Delta\right)=\kappa_{\sigma}\left(W, K_{W}+\Delta_{W}\right)
$$

by Lemma 2.7, and the divisor $G_{W}=g^{*} G^{\prime}+E_{W}-\Delta_{W}$ is Cartier. We have

$$
K_{W} \sim_{\mathbb{Q}} G_{W},
$$

and we claim that $G_{W} \geqslant 0$. Indeed, if $S^{\prime}$ is a component of $\Delta_{W}$, then $a\left(S^{\prime}, X^{\prime}, \Delta^{\prime}\right)=-1$. By Proposition 2.14, there exist a geometric valuation $S$ over $X$ and an integer $1 \leqslant r \leqslant m$ such that $\pi\left(c_{X^{\prime}}\left(S^{\prime}\right)\right)=c_{X}(S)$ and

$$
a\left(S^{\prime}, X^{\prime}, \Delta^{\prime}\right)+1=r(a(S, X, \Delta)+1) .
$$




\section{A note on the ABUndance CONJECTURE}

This implies $a(S, X, \Delta)=-1$; thus $c_{X}(S) \subseteq \operatorname{Supp} \Delta$ because $(X, \Delta)$ is log smooth. From here we obtain $c_{X^{\prime}}\left(S^{\prime}\right) \subseteq \pi^{-1}(\operatorname{Supp} \Delta)=\operatorname{Supp} G^{\prime}$, and in particular $S^{\prime} \subseteq \operatorname{Supp} g^{*} G^{\prime}$. Therefore mult $_{S^{\prime}} g^{*} G^{\prime} \geqslant 1=$ mult $_{S^{\prime}} \Delta_{W}$, and the claim follows.

Now, consider the klt pair $\left(K_{W}, \frac{1}{2} \Delta_{W}\right)$. Since $K_{W}+\frac{1}{2} \Delta_{W} \sim_{\mathbb{Q}} G_{W}+\frac{1}{2} \Delta_{W}, K_{W}+\Delta_{W} \sim_{\mathbb{Q}}$ $G_{W}+\Delta_{W}$ and $\operatorname{Supp}\left(G_{W}+\frac{1}{2} \Delta_{W}\right)=\operatorname{Supp}\left(G_{W}+\Delta_{W}\right)$, by Lemma 2.9 we have

$$
\kappa\left(X, K_{X}+\Delta\right)=\kappa\left(W, K_{W}+\frac{1}{2} \Delta_{W}\right) \quad \text { and } \quad \kappa_{\sigma}\left(X, K_{X}+\Delta\right)=\kappa_{\sigma}\left(W, K_{W}+\frac{1}{2} \Delta_{W}\right) .
$$

Let $k$ be the smallest positive integer such that $k\left(K_{W}-G_{W}\right) \sim 0$, and let $\mu: T \rightarrow W$ be the corresponding $k$-fold étale covering. Then

$$
K_{T}=\mu^{*} K_{W} \sim \mu^{*} G_{W} \geqslant 0,
$$

and setting $\Delta_{T}=\mu^{*}\left(\frac{1}{2} \Delta_{W}\right)$, the pair $\left(K_{T}, \Delta_{T}\right)$ is klt by Proposition 2.14. We have

$$
\kappa\left(X, K_{X}+\Delta\right)=\kappa\left(T, K_{T}+\Delta_{T}\right) \quad \text { and } \quad \kappa_{\sigma}\left(X, K_{X}+\Delta\right)=\kappa_{\sigma}\left(T, K_{T}+\Delta_{T}\right)
$$

by Lemma 2.9; hence $\kappa\left(X, K_{X}+\Delta\right)=\kappa_{\sigma}\left(X, K_{X}+\Delta\right)$ by our assumptions and by Lemma 2.8.

Remark 3.6. In the proof of Theorem 3.5, the inclusion $X^{\prime} \backslash \Delta^{\prime} \subseteq X^{\prime}$ is a toroidal embedding since the pair $(X, \Delta)$ is $\log$ smooth [Ara14, Lemma 1.1]; that is, it is locally analytically on $X^{\prime}$ isomorphic to an embedding of a torus into a toric variety. By [AW97, Theorem 0.2], there exists a toroidal resolution $h:\left(U, \Delta_{U}\right) \rightarrow\left(X^{\prime}, \Delta^{\prime}\right)$. Then we have $K_{U}+\Delta_{U}=h^{*}\left(K_{X^{\prime}}+\Delta^{\prime}\right)$. Indeed, locally in the analytic category both sides of this equation are trivial, which implies that all relevant discrepancies are zero. This is all implicit already in [KKMSD73]. The pair $\left(U, \Delta_{U}\right)$ is $\log$ smooth, and as in the proof of Theorem 3.5, one shows that $K_{U}$ is linearly equivalent to an effective Cartier divisor. Compare to [dFKX, Section 5].

Finally we can prove our main results.

Proof of Theorem 1.3. Let $(X, \Delta)$ be a uniruled klt pair. By replacing $(X, \Delta)$ by its terminal modification, see Theorem 2.2, we may assume that the pair $(X, \Delta)$ is terminal, and thus that $K_{X}$ is not pseudoeffective by Theorem 2.3. Let $\tau=\tau(X, 0 ; \Delta)=\min \left\{t \in \mathbb{R} \mid K_{X}+\right.$ $t \Delta$ is pseudoeffective . Since $K_{X}$ is not pseudoeffective and $K_{X}+\Delta$ is pseudoeffective, we have $0<\tau \leqslant 1$. If $\tau=1$, then we conclude by Theorem 3.3.

Therefore, we may assume $\tau<1$, and hence by Theorem 3.3 there exists a $\mathbb{Q}$-divisor $D_{\tau} \geqslant 0$ such that $K_{X}+\tau \Delta \sim_{\mathbb{Q}} D_{\tau}$. This yields

$$
K_{X}+\Delta \sim_{\mathbb{Q}} D \geqslant 0, \text { where } D=D_{\tau}+(1-\tau) \Delta .
$$

In particular, $\operatorname{Supp} \Delta \subseteq \operatorname{Supp} D$. Let $f: Y \rightarrow X$ be a log resolution of the pair $(X, D)$. Then we may write

$$
K_{Y}+\Gamma \sim_{\mathbb{Q}} f^{*}\left(K_{X}+\Delta\right)+E,
$$

where $\Gamma$ and $E$ are effective $\mathbb{Q}$-divisors with no common components, and $\Gamma=f_{*}^{-1} \Delta$ since $(X, \Delta)$ is a terminal pair. In particular, if we denote $D_{Y}=f^{*} D+E$, then $K_{Y}+\Gamma \sim_{\mathbb{Q}} D_{Y}$ and $\operatorname{Supp} \Gamma \subseteq \operatorname{Supp} D_{Y}$. We have

$$
\kappa\left(X, K_{X}+\Delta\right)=\kappa\left(Y, K_{Y}+\Gamma\right) \quad \text { and } \quad \kappa_{\sigma}\left(X, K_{X}+\Delta\right)=\kappa_{\sigma}\left(Y, K_{Y}+\Gamma\right)
$$

by Lemma 2.7 ; hence by replacing $(X, \Delta)$ by $(Y, \Gamma)$ and $D$ by $D_{Y}$, we may assume that $(X, D)$ is a $\log$ smooth pair. Finally, by replacing $\Delta$ by $\Delta+\varepsilon D$ for $0<\varepsilon \ll 1$, we may further assume $\operatorname{Supp} \Delta=\operatorname{Supp} D$. We conclude by Theorem 3.5 and by Lemma 2.8 . 


\section{T. Dorsch AND V. LAZIĆ}

Proof of Theorem 1.1. Let $(X, \Delta)$ be a uniruled klt pair. As in the proofs of Theorems 1.3 and 3.5, there exists a $\log$ smooth klt pair $\left(T, \Delta_{T}\right)$ such that $\left|K_{T}\right| \neq \emptyset$ and

$$
\kappa\left(X, K_{X}+\Delta\right)=\kappa\left(T, K_{T}+\Delta_{T}\right) \geqslant 0 \quad \text { and } \quad \kappa_{\sigma}\left(X, K_{X}+\Delta\right)=\kappa_{\sigma}\left(T, K_{T}+\Delta_{T}\right) .
$$

In particular, $T$ is not uniruled by Theorem 2.3. By Theorem 2.5, there exists a log terminal model $\left(T, \Delta_{T}\right) \rightarrow\left(T^{\prime}, \Delta_{T^{\prime}}\right)$ of $\left(T, \Delta_{T}\right)$; hence

$$
\kappa\left(T^{\prime}, K_{T^{\prime}}+\Delta_{T^{\prime}}\right)=\kappa_{\sigma}\left(T^{\prime}, K_{T^{\prime}}+\Delta_{T^{\prime}}\right),
$$

since we assume the abundance conjecture for non-uniruled pairs. We conclude by Lemmas 2.7 and 2.8 .

Proof of Theorem 1.2. The result is immediate from Theorem 1.3.

Remark 3.7. Assume that for every smooth variety of dimension $n$ with $K_{X}$ pseudoeffective we have $\kappa\left(X, K_{X}\right) \geqslant 0$. Then the previous proofs show that if good models exist for log smooth klt pairs $(X, \Delta)$ of dimension $n$ such that the linear system $\left|K_{X}\right|$ is not empty, then good models exist for klt pairs in dimension $n$.

Indeed, by Theorem 1.2 we only have to show that the assumptions imply the existence of good models for non-uniruled klt pairs in dimension $n$. Fix such a pair $(X, \Delta)$, and note that we may assume that the pair is terminal by Theorem 2.2. Then $\kappa\left(X, K_{X}\right) \geqslant 0$ by our assumption; hence there exists an effective divisor $D^{\prime}$ such that $K_{X} \sim_{\mathbb{Q}} D^{\prime}$. In particular, by denoting $D=D^{\prime}+\Delta$ we have $K_{X}+\Delta \sim_{\mathbb{Q}} D$ and $\operatorname{Supp} \Delta \subseteq \operatorname{Supp} D$. As in the proof of Theorem 1.3, by passing to a log resolution, we may assume that $(X, D)$ is log smooth. By replacing $\Delta$ by $\Delta+\varepsilon D$ for $0<\varepsilon \ll 1$, we may further assume $\operatorname{Supp} \Delta=\operatorname{Supp} D$, and we conclude by Theorem 3.5 and by Lemma 2.8.

This leads to the following result.

Lemma 3.8. Let $(X, \Delta)$ be a $\mathbb{Q}$-factorial terminal pair such that $\kappa\left(X, K_{X}\right) \geqslant 0$. Then there exist a generically finite morphism $f: Y \rightarrow X$ from a smooth variety $Y$ and an effective $\mathbb{Q}$-divisor $\Gamma$ on $Y$ with simple normal crossings support such that the pair $(Y, \Gamma)$ is $k l t,\left|K_{Y}\right| \neq \emptyset$ and

$$
\kappa\left(X, K_{X}+\Delta\right)=\kappa\left(Y, K_{Y}+\Gamma\right) \quad \text { and } \quad \kappa_{\sigma}\left(X, K_{X}+\Delta\right)=\kappa_{\sigma}\left(Y, K_{Y}+\Gamma\right) .
$$

If $\Delta=0$, we may additionally assume $\Gamma=0$.

Proof. The first claim follows from the proof of Theorem 1.3. When $\Delta=0$, as in Remark 3.7 we may assume that $X$ is smooth and that there exists a $\mathbb{Q}$-divisor $D^{\prime} \geqslant 0$ with simple normal crossings support such that $K_{X} \sim_{\mathbb{Q}} D^{\prime}$. Setting $\Delta_{X}=\varepsilon D^{\prime}$ and $D=D^{\prime}+\Delta_{X}$ for a rational number $0<\varepsilon \ll 1$, we have $K_{X}+\Delta_{X} \sim_{\mathbb{Q}} D$ and $0<$ mult $_{E} \Delta_{X}<$ mult $_{E} D$ for every component $E$ of $D$. Then with notation from the proof of Theorem 3.5, we obtain a generically finite map $\left(W, \Delta_{W}\right) \rightarrow\left(X, \Delta_{X}\right)$ such that the pair $\left(W, \Delta_{W}\right)$ is $\log$ smooth,

$$
\kappa\left(W, K_{W}+\Delta_{W}\right)=\kappa\left(X, K_{X}+\Delta_{X}\right) \quad \text { and } \quad \kappa_{\sigma}\left(W, K_{W}+\Delta_{W}\right)=\kappa_{\sigma}\left(X, K_{X}+\Delta_{X}\right),
$$

and $K_{W} \sim_{\mathbb{Q}} G_{W}$ for some Cartier divisor $G_{W}$ such that-crucially-Supp $G_{W}=\operatorname{Supp}\left(G_{W}+\right.$ $\left.\Delta_{W}\right)$. In particular, by Lemma 2.9 this implies

$$
\kappa\left(W, K_{W}\right)=\kappa\left(X, K_{X}\right) \text { and } \kappa_{\sigma}\left(W, K_{W}\right)=\kappa_{\sigma}\left(X, K_{X}\right) .
$$

Finally, one more étale cover allows us to conclude as in the proof of Theorem 3.5. 


\section{A Note ON THE ABUNDANCE CONJECTURE}

\section{ACKNOWLEDGEMEnTS}

We would like to thank $\mathrm{C}$. Xu for helpful discussions and comments about toroidal embeddings (see Remark 3.6), and O. Fujino, J. Kollár, S. Kovács, M. Mustaţă and Th. Peternell for useful conversations related to this work.

\section{REFERENCES}

Amb05 F. Ambro, The moduli b-divisor of an lc-trivial fibration, Compos. Math. 141 (2005), no. 2, 385-403; http://dx.doi.org/10.1112/S0010437X04001071.

Ara14 D. Arapura, Hodge theory of cyclic covers branched over a union of hyperplanes, Canad. J. Math. 66 (2014), no. 3, 505-524; http://dx.doi.org/10.4153/CJM-2013-040-8.

AW97 D. Abramovich and J. Wang, Equivariant resolution of singularities in characteristic 0, Math. Res. Lett. 4 (1997), no. 3, 427-433; http://dx.doi.org/10.4310/MRL.1997.v4.n3.a11.

BCHM10 C. Birkar, P. Cascini, C. D. Hacon and J. McKernan, Existence of minimal models for varieties of log general type, J. Amer. Math. Soc. 23 (2010), no. 2, 405-468; http://dx.doi.org/10. 1090/S0894-0347-09-00649-3.

BDPP13 S. Boucksom, J.-P. Demailly, M. Păun and Th. Peternell, The pseudo-effective cone of a compact Kähler manifold and varieties of negative Kodaira dimension, J. Algebraic Geom. 22 (2013), no. 2, 201-248; http://dx.doi.org/10.1090/S1056-3911-2012-00574-8.

Bir11 C. Birkar, On existence of log minimal models II, J. Reine Angew. Math. 658 (2011), 99-113; http://dx.doi.org/10.1515/CRELLE.2011.062.

CL12 P. Cascini and V. Lazić, New outlook on the minimal model program, I, Duke Math. J. 161 (2012), no. 12, 2415-2467; http://dx.doi.org/10.1215/00127094-1723755.

CL13 A. Corti and V. Lazić, New outlook on the minimal model program, II, Math. Ann. 356 (2013), no. 2, 617-633; http://dx.doi.org/10.1007/s00208-012-0858-1.

DHP13 J.P. Demailly, C.D. Hacon and M. Păun, Extension theorems, non-vanishing and the existence of good minimal models, Acta Math. 210 (2013), no. 2, 203-259; http://dx.doi.org/ 10.1007/s11511-013-0094-x.

EV92 H. Esnault and E. Viehweg, Lectures on vanishing theorems, DMV Seminar, vol. 20 (Birkhäuser Verlag, Basel, 1992); http://dx.doi.org/10.1007/978-3-0348-8600-0.

FG14 O. Fujino and Y. Gongyo, Log pluricanonical representations and the abundance conjecture, Compos. Math. 150 (2014), no. 4, 593-620; http://dx.doi.org/10.1112/ S0010437X13007495.

dFKX T. de Fernex, J. Kollár and C. Xu, The dual complex of singularities, arXiv:1212.1675.

Fuj05 O. Fujino, Addendum to: "Termination of 4-fold canonical flips" [Publ. Res. Inst. Math. Sci. 40 (2004), no. 1, 231-237], Publ. Res. Inst. Math. Sci. 41 (2005), no. 1; http://dx.doi. org/10.2977/prims/1145475410.

Fuj11_ On Kawamata's theorem, Classification of Algebraic Varieties, EMS Ser. Congr. Rep. (Eur. Math. Soc., Zürich, 2011), 305-315; http://dx.doi .org/10.4171/007-1/14.

GL13 Y. Gongyo and B. Lehmann, Reduction maps and minimal model theory, Compos. Math. 149 (2013), no. 2, 295-308; http://dx.doi.org/10.1112/S0010437X12000553.

Gon12 Y. Gongyo, Remarks on the non-vanishing conjecture, arXiv:1201.1128.

Har77 R. Hartshorne, Algebraic geometry, Grad. Texts in Math., vol. 52 (Springer-Verlag, New York - Heidelberg, 1977); http://dx.doi.org/10.1007/978-1-4757-3849-0.

HM10 C. D. Hacon and J. McKernan, Existence of minimal models for varieties of log general type. II, J. Amer. Math. Soc. 23 (2010), no. 2, 469-490; http://dx.doi.org/10.1090/ S0894-0347-09-00651-1. 


\section{T. Dorsch AND V. LAZIĆ}

HMX14 C. D. Hacon, J. McKernan and C. Xu, ACC for log canonical thresholds, Ann. of Math. 180 (2014), no. 2, 523-571; http://dx.doi.org/10.4007/annals.2014.180.2.3.

Kaw85a Y. Kawamata, Pluricanonical systems on minimal algebraic varieties, Invent. Math. 79 (1985), no. 3, 567-588; http://dx.doi.org/10.1007/BF01388524.

Kaw85b_ Minimal models and the Kodaira dimension of algebraic fiber spaces, J. Reine Angew. Math. 363 (1985), 1-46; http://dx.doi.org/10.1515/crll.1985.363.1.

Kaw92 A _ Abundance theorem for minimal threefolds, Invent. Math. 108 (1992), no. 2, 229-246; http://dx.doi.org/10.1007/BF02100604.

KKMSD73 G. Kempf, F. F. Knudsen, D. Mumford and B. Saint-Donat, Toroidal embeddings. I, Lecture Notes in Math., vol. 339 (Springer-Verlag, Berlin - New York, 1973); http://dx.doi.org/ 10.1007/BFb0070318.

KM98 J. Kollár and S. Mori, Birational geometry of algebraic varieties, Cambridge Tracts in Math., vol. 134 (Cambridge Univ. Press, Cambridge, 1998); http://dx.doi.org/10.1017/ CB09780511662560.

KMM94 S. Keel, K. Matsuki and J. McKernan, Log abundance theorem for threefolds, Duke Math. J. 75 (1994), no. 1, 99-119; http://dx.doi.org/10.1215/S0012-7094-94-07504-2.

Lai11 C.-J. Lai, Varieties fibered by good minimal models, Math. Ann. 350 (2011), no. 3, 533-547; http://dx.doi.org/10.1007/s00208-010-0574-7.

Leh13 B. Lehmann, Comparing numerical dimensions, Algebra Number Theory 7 (2013), no. 5, 1065-1100; http://dx.doi.org/10.2140/ant.2013.7.1065.

Miy87 Y. Miyaoka, The Chern classes and Kodaira dimension of a minimal variety, Algebraic Geometry (Sendai, 1985), Adv. Stud. Pure Math., vol. 10 (North-Holland, Amsterdam, 1987), 449-476.

Miy88a Abundance conjecture for 3-folds: case $\nu=1$, Compos. Math. 68 (1988), no. 2, 203-220; http://www. numdam.org/item?id=CM_1988_-68_2_203_0.

Miy88b_ On the Kodaira dimension of minimal threefolds, Math. Ann. 281 (1988), no. 2, 325-332; http://dx.doi.org/10.1007/BF01458437.

Mor88 S. Mori, Flip theorem and the existence of minimal models for 3-folds, J. Amer. Math. Soc. 1 (1988), no. 1, 117-253; http://dx.doi.org/10.2307/1990969.

Nak04 N. Nakayama, Zariski-decomposition and abundance, MSJ Memoirs, vol. 14 (Mathematical Society of Japan, Tokyo, 2004).

Rei80 M. Reid, Canonical 3-folds, Journées de Géometrie Algébrique d'Angers, Juillet 1979/Algebraic Geometry (Angers, 1979) (Sijthoff \& Noordhoff, Alphen aan den Rijn-Germantown, Md., 1980), 273-310.

Sho86 V. V. Shokurov, A nonvanishing theorem, Math. USSR Izv. 49 (1986), no. 3, 591-604; http: //dx.doi.org/10.1070/IM1986v026n03ABEH001160.

Tobias Dorsch dorsch@math.uni-bonn.de

Mathematisches Institut, Universität Bonn, Endenicher Allee 60, 53115 Bonn, Germany

Vladimir Lazić lazic@math.uni-bonn.de

Mathematisches Institut, Universität Bonn, Endenicher Allee 60, 53115 Bonn, Germany 